\title{
Removal of organic dyes from aqueous solutions with surfactant-modified magnetic nanoparticles
}

\author{
Katarzyna Wybieralska, Anna Wajda \\ Poznan University of Economics, Faculty of Commodity Science, Department of Technology and Instrumental Analysis, \\ al. Niepodległości 10, 61-875 Poznań, Poland \\ "Corresponding authors: e-mail: k.wybieralska@ue.poznan.pl
}

\begin{abstract}
The paper presents the results of studies on the possibility of using magnetic nanoparticles modified with selected hydrophobic surfactants for model post-production water purification. Colloidal solutions of iron hydroxide (III) and iron oxide (II and III) were obtained and their particles were subjected to surface modification using surfactants. Thus obtained magnetic fluids were used as active agents in the process of removing selected organic dyes from their aqueous solutions. The effectiveness of the modified compounds was analysed using spectrophotometric methods. It has been shown that the effectiveness of the process depends on the type of surfactant used to modify selected magnetic nanoparticles.
\end{abstract}

Keywords: organic dyes, iron compounds, magnetic fluids, surfactants, ecology.

\section{INTRODUCTION}

In order to improve the quality of water, various desalination and purification methods are used, with particular emphasis on water intended for human consumption. These methods include, inter alia, the use of the phenomena of adsorption, biodegradation, photodegradation, catalysis, photocatalysis, etc. Most of them are based on the use of advanced materials, such as: oxidants $^{1}$, microbiological substances ${ }^{2}$, porous materials ${ }^{3}$, polymer membranes ${ }^{4}$, inorganic and organic flocculants ${ }^{5}$, photocatalysts ${ }^{6}$, etc. Significant progress in the field of water treatment is associated with the preparation of new materials and the pursuit of new ideas.

The presence of dyes in water is a serious problem for many industries, especially pulp and paper, dyestuffs, textiles and cosmetics. Even small amounts of dyes (of the order of several ppm) are undesirable. They cause discolouration of water, give it an unsightly appearance and disrupt life processes that occur there ${ }^{7}$. Most dyes are not biodegradable, reduce light penetration into water and inhibit the processes of photosynthesis.

Due to their unique properties, porous materials, especially porous carbons (e.g. activated carbons, microporous activated carbon fibres) and functional polymers are widely used in industrial processes of removing impurities from water and $a_{i}{ }^{8}$. These compounds can be effectively used in purification processes due to their high surface area, high porosity, slight chemical activity and high mechanical strength.

One of the most interesting water treatment methods involves using the effect of magnetic field on a flowing liquid stream. So far, a magnetic field has been used to remove limescale deposits from vessels. Here, it is necessary to use appropriate magnetizers. Easy application and environmental neutrality are the advantages of the method.

The effect of magnetic field can also be successfully used to remove organic contaminants such as dyes.

\section{Magnetic liquids}

Magnetic fluids or ferrofluids are colloidal suspensions of magnetically active particles in non-magnetic carrier liquid. The average size of the particles is $10 \mathrm{~nm}$. Gen- erally, magnetic fluid comprises $5 \%$ of ferromagnetic particles, $10 \%$ of surfactants and $85 \%$ of carrier fluid'.

Magnetic fluids are used among others as active vibration damping or sealing substances ${ }^{\mathbf{1 0}-11}$. In addition, they are used in recycling for separation of metal pieces, and in various devices for noise reduction. Next to the technological applications, they are widely used in medicine (e.g. blocking the blood supply to tumour cells or treatment by local, artificial increase in temperature).

This paper presents the test method for the synthesis of magnetic nanoparticle systems, based on a magnetite structure modified with selected surfactants. Surfactants are substances which are soluble both in polar and non-polar fluids due to their amphiphilic properties resulting from their characteristic asymmetric chemical structure. This asymmetric structure determines the behaviour in relation to different phases constituting the system, which in turn translates into the arrangement of the surfactant molecule and determines the surface activity manifested by decreased surface tension of the liquid. The structure of surfactants also determines the possibility of their adsorption onto the particles of other substances of opposite surface charge.

Apart from surfactants, the studies used the colloidal solution of iron hydroxide (III) and iron oxide (II, III). The surface of the thus defined magnetic core of the particle was chemically modified using long-chain surfactants and the sorption properties of the resulting magnetic fluid particles were described. The possibility of their use for the purification of water containing selected organic dyes as model pollutants was also evaluated.

\section{EXPERIMENTAL PART}

The main objective of the studies was to determine the sorption properties of the synthesized particles and check the possibility of using thus obtained magnetic nanoparticles for the purification of water containing selected organic dyes. In order to quantify the effectiveness of the compounds obtained, the removal degree of dye molecules by the obtained magnetic fluids modified with selected surfactants was evaluated using a spectrophotometric method. The measurement of decrease in absorbance of model dye solution treated 
with magnetic nanoparticles and the external magnetic field was analysed.

The results obtained refer to the preparation of magnetic nanoparticle systems, based on the structure of magnetite $\left(\mathrm{Fe}_{3} \mathrm{O}_{4}\right)$ and iron hydroxide (III), the surface of which was modified with selected anionic and cationic surfactants. The reaction resulted in adsorbents the task of which was to neutralize the selected organic dyes: methyl orange, crystal violet, Congo red and bromothymol blue and methylene blue.

\section{MATERIALS}

Sodium dodecyl sulfate (SDS, Merck), bis-(2-ethylhexyl) sodium sulfosuccinate (AOT, Sigma), hexadecyltrimethylammonium bromide, CTAB (Tokyo Kogyo Kassei) hexadecylpyridinium chloride, CPC (Merck), sodium hydroxide (POCh Gliwice) chloride iron (III) (POCh Gliwice), isopropanol (POCh Gliwice), methyl orange (POCh Gliwice), Congo Red (POCh Gliwice), Mohr's salt (POCh Gliwice), bromothymol blue (POCh Gliwice), methylene blue (POCh Gliwice), crystal violet (POCh Gliwice).

Iron oxide (II, III) is a substance with magnetic properties which occurs in nature as a mineral called magnetite. This oxide crystallizes in a regular system, producing black spinel crystal structures. It is characterized by ferrimagnetic properties. Iron hydroxide (III) exists in nature in a partially dehydrated form as goethite and lepidolite. Due to its characteristics, it is used for water purification.

\section{Preparation of magnetic nanoparticles}

Iron hydroxide (III) was prepared according to the following equations:

$\mathrm{FeCl}_{3}+3 \mathrm{H}_{2} \mathrm{O} \rightarrow \mathrm{Fe}(\mathrm{OH})_{3}+3 \mathrm{HCl}$

$\mathrm{Fe}(\mathrm{OH})_{3}+\mathrm{HCl} \rightarrow \mathrm{FeOCl}+2 \mathrm{H}_{2} \mathrm{O}$

$\mathrm{nFe}(\mathrm{OH})_{3}+\mathrm{mFeO}^{+}+\mathrm{mCl}^{-} \rightarrow \mathrm{nFe}(\mathrm{OH})_{3} \cdot \mathrm{mFeO}^{+}$ $+\mathrm{mCl}$

In order to obtain magnetic nanoparticles comprising iron oxide (II, III), the following synthesis was performed $^{\mathbf{1 2}}$ :

$\mathrm{FeCl}_{2}+2 \mathrm{FeCl}_{3}+8 \mathrm{NH} 3+4 \mathrm{H}_{2} \mathrm{O} \rightarrow \mathrm{Fe}_{3} \mathrm{O}_{4}+8 \mathrm{NH}_{4} \mathrm{Cl}$

These solutions of iron hydroxide (III) and iron oxide (II and III) were washed several times with redistilled water, using the external magnetic field produced by neodymium magnets to separate the product from the post-reaction mixture (Fig. 1).

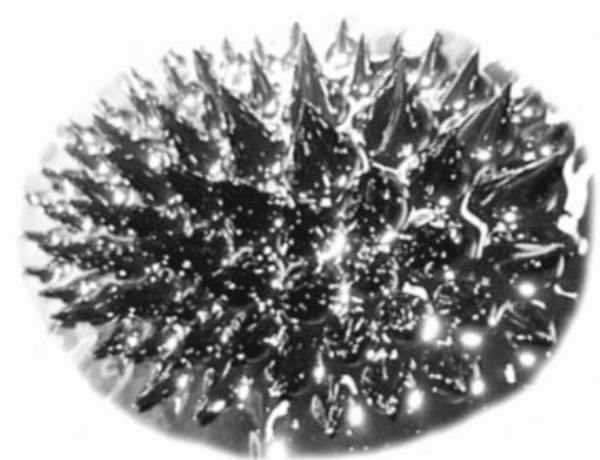

Figure 1. Ferrofluid in the magnetic field, an example: a system of SDS-modified iron oxide (II and III) in a solution with the addition of OM

\section{Surface modification with surfactants}

Basic solutions of respectively synthesized iron oxide (II and III) and iron hydroxide (III) were modified with specific surfactants.

Suitable proportions of the solutions of $\mathrm{Fe}_{3} \mathrm{O}_{4}$ and iron hydroxide (III) suspension (for example, in a volume ratio of 1:2) were mixed with solutions of selected surfactants. As a result, a crude product in the form of sludge was obtained. In order to remove unreacted surfactant particles, the resulting post-reaction mixture was washed repeatedly with redistilled water. The modification resulted in products in powder form (Figs. 2 and $3)$. The reaction product obtained was in gel form only after the modification with bis(2-ethylhexyl) sodium sulfosuccinate (AOT). In further studies, these products were used without undergoing additional modifications of their physical form.

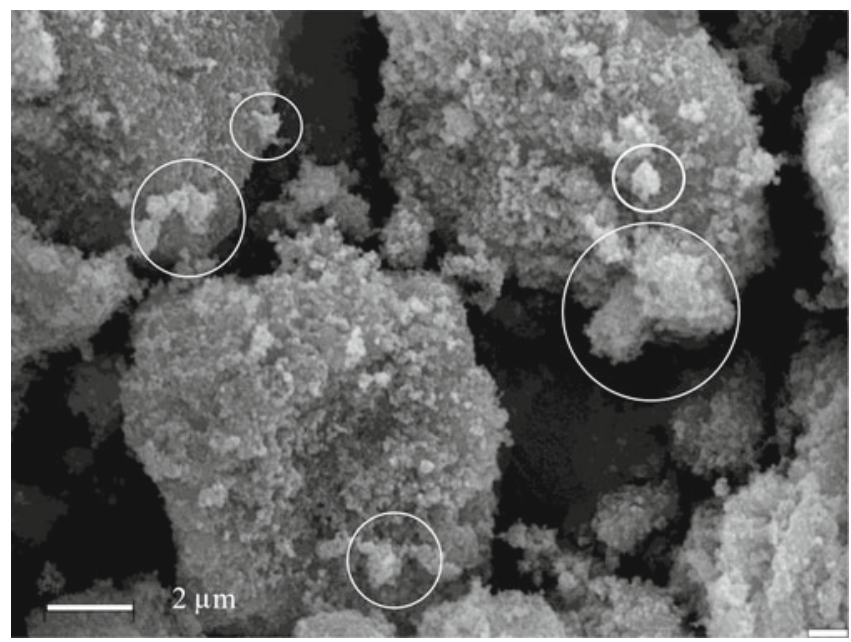

Figure 2. SEM image of iron hydroxide (III) modified with sodium dodecyl sulfate (SDS). The image indicates probable positions of the surfactant

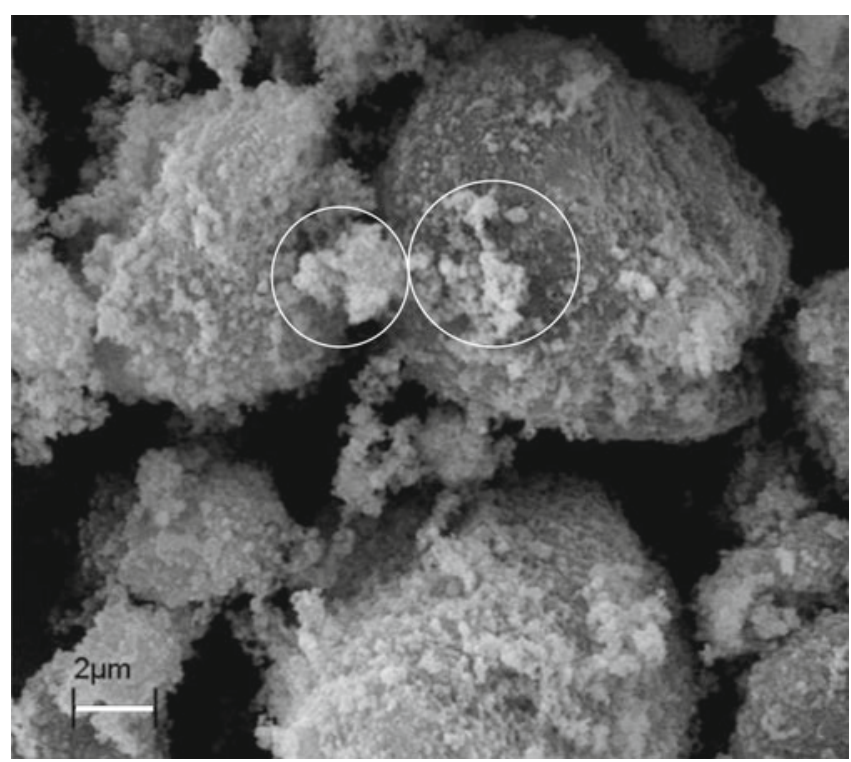

Figure 3. SEM image of $\mathrm{Fe}_{3} \mathrm{O}_{4}$ modified with sodium dodecyl sulfate (SDS). The image indicates the attached surfactant

The adsorption properties of the modified magnetic nanoparticles obtained were tested in the model systems of organic dye solutions, the concentrations of which were chosen to show individual absorbance values. In order to determine the removal efficiency of dyes tested 
from their aqueous solutions, a change in dye concentration by the action of the modified iron compounds was determined using a spectrophotometric method. Absorbance measurements were performed using a Genesys II spectrophotometer (Milton Roy) at intervals of $5 \mathrm{~min}$ for $60 \mathrm{~min}$. Kinetic studies were carried out at wavelengths corresponding to the maximum absorption of dyes studied.

\section{RESULTS}

In order to quantify the removal efficiency of dyes tested from their aqueous solutions with the surface-modified iron hydroxide (III), the concept of a relative percentage efficiency coefficient $(\mathrm{E})$ of removing individual dyes was introduced and defined by the equation:

$E=\frac{\left(A_{0}-A_{1}\right)}{A_{0}} \cdot 100 \%$

where:

$\mathrm{A}_{0}$ - initial absorbance of the test dye solution (before the process of adsorption),

$\mathrm{A}_{1}$ - absorbance of the test dye solution determined after treatment with modified iron hydroxide (III).

The study of adsorption of methyl orange and bromothymol blue from their aqueous solutions using iron hydroxide (III) modified with sodium dodecyl sulphate showed a correlation of the amount of adsorbed molecules and the amount of adsorbent - the maximum values were: $89 \%$ for bromothymol blue, $43 \%$ for crystal violet and $63 \%$ for methyl orange. The adsorption of Congo red molecules showed no dependence on the amount of adsorbent and remained at an average of 58\% (Table 1).

The studies on the use of bis(2-ethylhexyl) sodium sulfosuccinate (AOT) for surface modification of iron hydroxide (III) have shown that this method of surface modification is less efficient. Lower reduction of dye concentration was obtained than in the case of iron hydroxide (III) modified with sodium dodecyl sulfate. Reduction degrees for the modification with AOT are as follows: $15 \%$ for methyl orange, $26 \%$ for bromothymol blue, $11 \%$ for crystal violet and $60 \%$ for Congo red.

The results of absorbance measurements of dyes treated with magnetic fluids modified with surfactants made it possible to determine the process efficiency using iron oxide (II, III). A relative change in the absorbance of dye solution by the action of iron oxide (II, III) modified with surfactants was analysed. The adsorbent mass was $0.1 \mathrm{~g}$ and the volume of the purified system was $15 \mathrm{~cm}^{3}$.

Figure 4 shows the kinetics of purification of aqueous solutions of methyl orange, crystal violet and methylene blue using magnetic nanoparticles modified with the

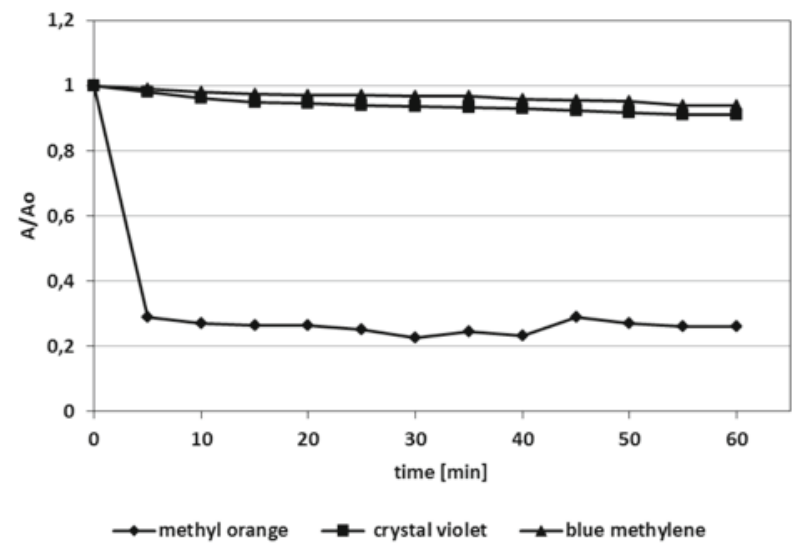

Figure 4. Kinetics of dye adsorption from aqueous solutions using $\mathrm{Fe}_{3} \mathrm{O}_{4}$ nanoparticles modified with $\mathrm{CTAB}$

cationic surfactant. The largest decrease in relative absorbance was observed for methyl orange. The anionic dye is removed most efficiently by the cationic surfactant. In the case of removing dyes with anionic surfactant-modified particles, a decrease in absorbance was observed for crystal violet and methylene blue.

The studies have shown that iron oxide (II and III) modified with the anionic surfactant - sodium dodecyl sulfate is the most effective (Fig. 5). Relative absorbance decreased most significantly when removing the cationic dyes - crystal violet and methylene blue (relative absorbance decreased during the first 15 minutes of measurement).

The quantitative analysis of dye adsorpted from the aqueous solution by magnetic liquid modified with selected surfactants is presented as a dye concentration change

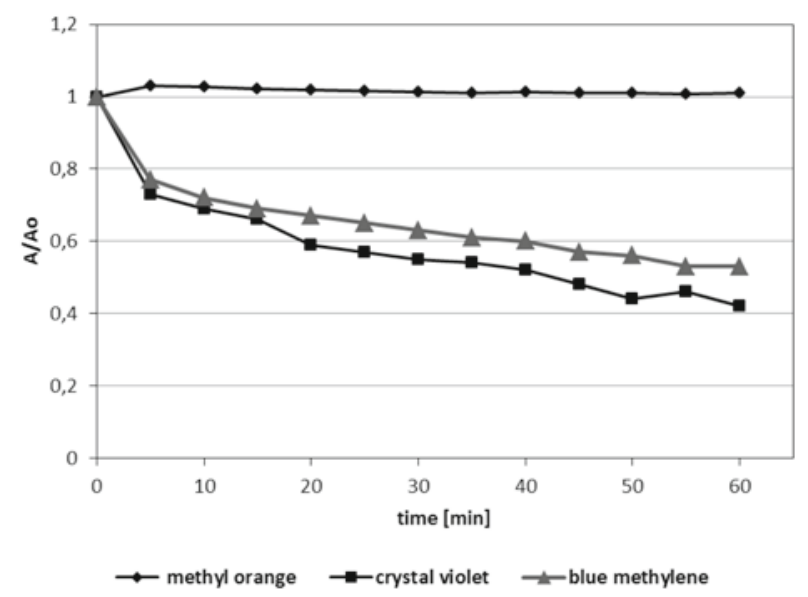

Figure 5. Kinetics of dye adsorption from aqueous solutions using $\mathrm{Fe}_{3} \mathrm{O}_{4}$ nanoparticles modified with SDS

Table 1. Effect of iron hydroxide (III) modified with surfactants on the removal efficiency of selected dyes from model solutions. The volume of the treated system is $6 \mathrm{~cm}^{3}$

\begin{tabular}{|c|c|c|}
\hline Adsorbed dye /(concentration [M]) & Adsorbent mass [g] & Dye removal efficiency [\%] \\
\hline \multicolumn{3}{|c|}{$\mathrm{Fe}(\mathrm{OH})_{3}-\mathrm{SDS}$} \\
\hline Bromothymol blue $\left(8.3 \cdot 10^{-5}\right)$ & 0.08 & 89 \\
\hline Methyl orange $\quad\left(1.8 \cdot 10^{-5}\right)$ & 0.015 & 63 \\
\hline Crystal violet & $0.02-0.1$ & 43 \\
\hline Congo red $\quad\left(7.4 \cdot 10^{-5}\right)$ & 0.02 & 58 \\
\hline \multicolumn{3}{|c|}{$\mathrm{Fe}(\mathrm{OH})_{3}-\mathrm{AOT}$} \\
\hline Bromothymol blue $\left(8.3 \cdot 10^{-5}\right)$ & 0.09 & 26 \\
\hline Methyl orange $\quad\left(1.8 \cdot 10^{-5}\right)$ & 0.09 & 15 \\
\hline Crystal violet $\quad\left(1.0 \cdot 10^{-6}\right)$ & 0.01 & 11 \\
\hline Congo red $\quad\left(7.4 \cdot 10^{-5}\right)$ & 0.08 & 60 \\
\hline
\end{tabular}




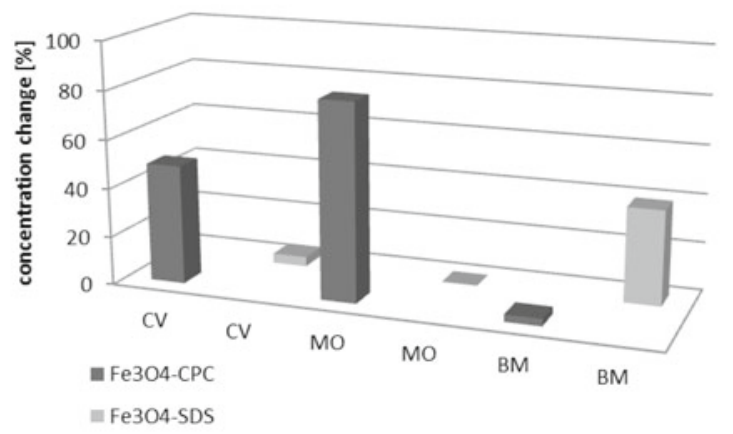

Figure 6. Value of dye absorbed from the aqueous solution - CV (crystal violet), MO (methyl orange), BM (methylene blue) by magnetic liquid modified with surfactants

after 60 minutes of measurement. Figure 6 presents the activity of iron oxide (II and III) modified with the cationic surfactant - cetyl pyridinium chloride (CPC) and the anionic surfactant - sodium dodecyl sulfate (SDS). The experiments showed that SDS removed half (49\%) of the crystal violet molecules from the solution. The effectiveness of CPC was $4 \%$. The concentration of methyl orange bound to the magnetic fluid modified with CPC was $80 \%$. When using SDS, no dye molecule adsorption was observed. Iron oxide (II and III) modified with SDS gave the best results when removing methylene blue from the aqueous solutions $-40 \%$. The CPC-modified magnetic fluid allowed the capture of as little as $3 \%$ of the dye molecules from the solution.

\section{CONCLUSIONS}

The iron compounds: iron oxide (II, III) iron hydroxide (III) treated with selected surfactants were used to test their ability to purify water contaminated with organic dyes. The conducted experiments made it possible to determine a correlation of relative absorbance and time. The kinetics of binding dye molecules by magnetic liquid modified with selected surfactants was determined. It has been found that the efficiency of water purification using magnetic nanoparticles tested depends on the type of surfactant and the chemical structure of dye. It has been shown that methyl orange is much more efficiently adsorbed from aqueous solutions by iron oxide (II and III) modified with the cationic surfactants than crystal violet and methylene blue. Molecules of methylene blue and crystal violet are effectively captured by magnetic fluid modified with the anionic surfactant. The subject of subsequent studies should be to determine the effect of charge of the active part of surfactant or dye and the possibility of interactions between the aromatic rings of surfactants and dyes on the kinetics and efficiency of the adsorption process in these systems.

The efficiency of purification of the model dye solutions using iron hydroxide (III) modified with surfactants also depends on the type of surfactant used. Higher dye adsorption efficiency was demonstrated when using sodium dodecyl sulfate (SDS).

The studies confirm the effectiveness of the water purification method using iron hydroxide (III) modified with surfactants as a potential substance removing organic dyes from water reservoirs.

\section{LITERATURE CITED}

1. Oxidation technologies for water and wastewater treatment IV (2007), Water Science and Technology 55, 12.

2. Mara, M. \& Horan, N. (2003). The Handbook of Water and Wastewater Microbiology. Academic Press, London.

3. Bruce, D.W., Walton, R. \& O'Hare, D. (2010). Porous Materials. John Wiley \& Sons Inc., London.

4. Marchand-Brynaert, J. (2011). Polymer Membranes, in: P. Somasundaran, [Ed.] A. Hubbard: Encyclopedia of Surface and Colloid Science. Taylor and Francis, London.

5. Heitner, H.I. (2004). Flocculating agents, in: Kirk-Othmer, Encyclopedia of Chemical Technology. John Wiley \& Sons Inc., London.

6. Bodzek, M. \& Rajca, M. (2012). Photocatalysis in the treatment and disinfection of water. Part I. Theoretical backgrounds. Ecological Chemistry and Engineering S. 19(4), 489-512.

7. Robinson, T., Chandran, B. \& Nigam, P. (2002). Removal of dyes from a synthetic textile dye effluent by biosorption on apple pomace and wheat straw. Water Research, 36, 2824-2830. DOI: 10.1016/S00431354(01)00521-8.

8. Chen, K.C., Wu, J.Y., Huang, C.C., Liang, Y.M. \& Hwang, S.C.J. (2003). Decolorization of azo dye using PVA-immobilized microorganisms. Journal of Biotechnology, 101, 241-252.

9. Filipcsei, G., Feher, J. \& Zrınyi, M. (2000). Electric field sensitive neutral polymer gels. Journal of Molecular Structure 554, 109-117.

10. Milecki, A. (2005). Basics of designing devices with magnetorheological fluids [in Polish]. Przegląd Mechaniczny 2, 29-33.

11. An-Hui, L., Salabas, E.L. \& Schuth, F. (2007). Magnetic Nanoparticles: Synthesis, Protection, Functionalization, and Application, Angewandte Chemie International Edition, 46, 1222-1244.

12. Zieliński, R., Wajda, A. \& Wybieralska, K. (2010). Use of cationic surfactants in surface modification of magnetic nanoparticles for adsorption of organic dyes from aqueous solutions [in Polish]. Przemyst Chemiczny 89 /11, 1264-1268. 